\title{
Microbial Diversity in Cultivated and Feral Vanilla Vanilla Planifolia Orchids Affected by Stem and Rot Disease
}

\author{
Ireri A. Carbajal-Valenzuela
}

CINVESTAV: Centro de Investigacion y de Estudios Avanzados del Instituto Politecnico Nacional Ariel H. Muñoz-Sanchez

CINVESTAV: Centro de Investigacion y de Estudios Avanzados del Instituto Politecnico Nacional Juan Hernández-Hernández

Instituto Nacional de Investigaciones Forestales

\section{Francisco Barona-Gómez}

CINVESTAV: Centro de Investigacion y de Estudios Avanzados del Instituto Politecnico Nacional

\section{Camille Truong}

Royal Botanic Gardens Victoria, Birdwood Avenue, Melbourne

Angelica Cibrian-Jaramillo ( $\nabla$ angelica.cibrian@cinvestav.mx )

CINVESTAV: Centro de Investigacion y de Estudios Avanzados del Instituto Politecnico Nacional https://orcid.org/0000-0002-7974-455X

\section{Research Article}

Keywords: Fusarium, meta-barcoding, microbiome, pathogen resistance, Vanilla planifolia

Posted Date: July 13th, 2021

DOl: https://doi.org/10.21203/rs.3.rs-694307/v1

License: (c) (i) This work is licensed under a Creative Commons Attribution 4.0 International License. Read Full License 


\section{Abstract}

The worldwide production of vanilla, a native orchid from Mexico, is greatly affected by stem and root rot disease, typically associated with Fusarium oxysporum fungi. We hypothesized that the presence of Fusarium species in vanilla is not sufficient for the plant to express symptoms of the disease. We described the taxonomic composition of endophytic microbiomes in symptomatic, asymptomatic vanilla plants using 16S and ITS rDNA metabarcoding, and ITS Sanger sequences generated from fungal isolates. We compared the bacterial and fungal diversity in vanilla plants from a long-term plantation, and from feral plants found near abandoned plantations that did not present SRD symptoms. No significant differences were found in the species richness of the bacterial and fungal microbiome among feral, or asymptomatic and symptomatic cultivated vanilla. However, significant differences were detected in both fungal and bacterial diversity from different organs in the same plant, with roots being more diverse than stems. We found that Proteobacteria and Actinobacteria, as well as the fungal families Nectriaceae and Xylariaceae, constitute the core of the vanilla microbiome that inhabits the root and stem of both cultivated and feral plants. Our work provides information on the microbial diversity found in root and stem rot in vanilla and lays the groundwork for a better understanding of the role of the microbiome in fungal diseases in vanilla cultivation.

\section{Introduction}

Vanilla planifolia Jacks. ex Andrews, is an orchid species endemic to Mexico. Despite its remarkable historical, cultural, and socioeconomic value [1], Mexican vanilla consists of a mere $5 \%$ of the world's production, compared to $35 \%$ from Indonesia or $32 \%$ from Madagascar [2]. Low yields of Mexican vanilla and worldwide, are in part due to the plants' susceptibility to stem and root rot disease (SRD) [3]. When affected by SRD, vanilla plants show signs of necrosis until they rot [4]. It is estimated that $67.4 \%$ of vanilla plants are removed from Mexican plantations within 4 years of their initial establishment due to SRD [5]. SRD in vanilla has been associated with fungal species from the genus Fusarium [3,6,7], and specifically, with F. oxysporum f. sp. vanillae [8-10]. However, F. oxysporum and other species from this genus have been identified in both wild and cultivated $V$. planifolia plants, including those without SRD symptoms $[6,11,12]$. In fact, several Fusarium species, including some $F$. oxysporum strains can act as mutualistic microbes in plants, providing the inhibition of pathogenic strains and other benefits to their host [13]. This suggests a natural symbiotic role of Fusarium species in the vanilla microbiome and that its presence or abundance on vanilla plants is not sufficient to predict disease outcome.

Bacterial and fungal microbiomes can affect the development of SRD symptoms. For example, the Vanilla endophytic bacteria Bacillus amyloliquefaciens can inhibit the growth of pathogenic fungi in vitro [14]. Other studies show the correlation of soil microbial communities with the incidence of SRD in $V$. planifolia plantations; Xiong, et al. [15] found that disease incidence was positively correlated with higher bacterial diversity and lower fungal diversity, and negatively with lower bacterial diversity and higher fungal diversity in soil of vanilla plantations. Also, they found higher dominance of Mortierella spp. in soils with plants that present a low incidence of symptoms of SRD disease. Later, these authors 
demonstrated that the application of bio-fertilizers enhanced bacterial diversity and was a key factor for controlling F. oxysporum populations [16]. As a result of bio-fertilizer application, they observed an increase of microbial species with antifungal activity (Lysobacter spp.) in treated soils and suggested that SRD suppression was linked to the introduction of keystone species (Bacillus and Trichoderma spp.) that reshaped the soil microbiome structure and functions, indirectly inhibiting pathogens.

In this study we compared the bacterial and fungal microbiome of asymptomatic and symptomatic cultivated vanilla plants from long-term plantations, as well as from feral plants found near abandoned plantations that did not present SRD symptoms. Although these feral plants may be remnants of wild populations, we assumed that they most likely escaped from abandoned plantations and that have not been managed for several decades, with a history of natural pollination and biotic interactions. We hypothesized that 1 ) The diversity and community composition of the vanilla microbiome is different between feral and cultivated plants, with feral plants being more diverse; 2) Fusarium species occur in both symptomatic and asymptomatic plants, but that plants with SRD symptoms have higher abundances of Fusarium species; and that 3) Microbial diversity is the same in affected plant organs, mainly stem and root. Our research contributes to our understanding of the microbial diversity and functions in wild and cultivated vanilla populations and to develop methods to control the expression of stem and root disease.

\section{Methods}

Sampling. Sampling was carried out during August 2018 in a vanilla plantation near Papantla (20.4272696, -97.3136406), in the state of Veracruz, Mexico. The root and stem of six symptomatic individuals (with clear signs of SRD, such as chlorosis and necrosis) and six asymptomatic (no signs of SRD) individuals were sampled, for a total of 24 samples. For each sample, $10 \mathrm{~cm}$ of tissue was placed in a Ziploc bag and kept at $4{ }^{\circ} \mathrm{C}$ for less than $24 \mathrm{~h}$ before surface sterilization, following the procedure described by Pinaria et al [6] and fungal isolation in culture media. Additionally, the root and stem of 3 symptomatic, 3 asymptomatic and 2 feral plants growing in the tropical native forest surrounding the plantation, were preserved in liquid nitrogen for DNA extraction and meta-barcoding, for a total of 16 samples.

Fungal culturing. Approximately $1 \mathrm{~cm}$ was removed at both extremities of the samples to avoid microbiological bias from surface sterilization. The remaining material was cut open longitudinally and macerated in $10 \mathrm{~mL}$ of sterilized distilled water. $1 \mathrm{~mL}$ of a 1:100 dilution was uniformly plated using 3 commercial media: PDA, Czapek and Sabourad agar. Each media was prepared (1) following the manufacturer instructions and (2) by adding $300 \mathrm{~mL}$ of autoclaved vanilla macerated tissue to $700 \mathrm{~mL}$ of $0.5 \times$ agar. A total of 6 cultures per sample were maintained at room temperature in the dark for 6 days and axenic cultures were isolated into new PDA plates. In some cases, antibiotics (penicillin $200 \mathrm{uL} / \mathrm{mL}+$ streptomycin $100 \mathrm{uL} / \mathrm{mL}$ ) were added to avoid contamination with bacterial growth. 
Molecular identification of fungal isolates. Fungal axenic cultures were grouped into morphotypes and DNA was extracted from one isolate of each morphotype group following a standard CTAB-SDS protocol $[17,18]$. The internal transcribed spacer (ITS) region of rDNA was amplified with primers ITS1F [19] and ITS4 [14] using de GoTaq Master Mix from Promega and the following PCR conditions: initial denaturation at $95^{\circ} \mathrm{C}$ for $5 \mathrm{~min}$, followed by 32 cycles at $94^{\circ} \mathrm{C}$ for $30 \mathrm{~s}, 52^{\circ} \mathrm{C}$ for $30 \mathrm{~s}$, and $72{ }^{\circ} \mathrm{C}$ for 1 min, with a final extension step at $72^{\circ} \mathrm{C}$ for 8 min.

Amplicons were verified on $1.5 \%$ agarose gel and purified as follow: 1/10 volume of ammonium acetate $\left(\mathrm{NH}_{4} \mathrm{Ac}\right)$ and 2 volumes of ice-cold isopropanol were added to the PCR reaction, vortexed and kept at -20 ${ }^{\circ} \mathrm{C}$ for $1 \mathrm{~h}$. After centrifugation at $13500 \mathrm{rpm}$ during $30 \mathrm{~min}$ at $4^{\circ} \mathrm{C}$, the pellet was decanted and cleaned with cold 70\% isopropanol, then resuspended in DNA-free sterilized water. Once purified, amplicons were sequenced by Sanger sequencing in the Laboratorio Nacional de Genómica para la Biodiversidad at CINVESTAV, Mexico. DNA sequences were verified and assembled using Geneious 11.1.

Fungal isolates were taxonomically identified by performing BLAST searches to corresponding "Species Hypothesis" (SH) in the UNITE database [21]. SH are based on dynamic values of the identity percentage of the ITS region from $97 \%$ to $99.5 \%$; in this case, we assigned a SH to each sequence based on $97 \%$ identity. To further delimit species, phylogenetic trees were reconstructed for the most diverse taxonomic groups (Fusarium and Xylariaceae) with a representation of all reported species for which ITS was available in GenBank. Alignments were performed in Muscle [22], verified with Gblocks [23] with default parameters and used to generate maximum likelihood trees in MegaX [24] using the Tamura-Nei substitution model (selected based on the lowest AIC scores) and 1000 bootstrap replicates. Finally, matches between fungal isolates and OTUs generated in the meta-barcoding analysis were explored using BLAST searches, by selecting matches with $>97 \%$ identity and $>250 \mathrm{pb}$ sequence length.

Root and stem meta-barcoding. DNA was extracted using an optimized CTAB method [25] with root samples previously washed with a Sorbitol Buffer [26]. The bacterial 16S rRNA region V3-V4 was amplified using primers Bakt_341F and Bakt_805R [27] and the fungal region ITS2 with primers 3F and 4R [20]. Amplicon libraries were constructed and sequenced at Macrogen Inc. (Seul, South Korea), using an Illumina MiSeq $2 \times 300$ bp platform, with the following PCR conditions for both regions: initial denaturation at $95^{\circ} \mathrm{C}$ for $5 \mathrm{~min}$, followed by 25 cycles at $95^{\circ} \mathrm{C}$ for $30 \mathrm{~s}, 55^{\circ} \mathrm{C}$ for $30 \mathrm{~s}$, and $72{ }^{\circ} \mathrm{C}$ for 30 sec, with a final extension step at $72^{\circ} \mathrm{C}$ for $5 \mathrm{~min}$.

Sequence quality filtering and clustering into "Operational Taxonomic Units" (OTU) at 97\% similarity was carried out in AMPtk [28] with default parameters except: For quality-trimming: -min_len 150 as the minimum length to keep a sequence and $-\mathrm{m} 10$ as the minimum number of reads per OTU; for filtering against index-bleed: -min_reads_otu 10 as the minimum number of reads for an OTU to be retained as valid. OTU taxonomy was assigned in AMPtk by aligning reference sequences in RDP (version V16) [29] for $16 \mathrm{~S}$ and UNITE (version 8.2) [21] for ITS2. Dubious OTUs with abundance $>0.05 \%$ of total read count per sample were filtered out, as well as OTUs that did not classify as bacteria or fungi. OTUs of 
mitochondrial and chloroplast origin were further filtered from the bacterial dataset by using the filter_taxa_from_otu_table.py function in QIIME [30].

Taxonomic diversity analyses were carried out in R 4.0.3 (R Core Development Team 2008) using custom scripts and the phyloseq, vegan, metacoder and ggplot2 packages [31-34] According to Fernandez et al. (2017) [35], we transformed read counts into relative abundances by averaging the number of reads per sample, multiplied by 10,000 and transformed to the next integer to be used as counts. Species richness (observed richness and Shannon index) between organ (root or stem) and conditions (symptomatic, asymptomatic, feral) were tested using non-parametric Kruskal-Wallis tests and pairwise Wilcoxon rank sum test. Bonferroni corrections were applied to $p$-values to account for multiple comparisons. Since read counts are nor representative of species abundances [36,37], we constructed Raup-Crick dissimilarity matrices based on OTU presence/absence (after eliminating OTUs only present in one sample) and visualized bacterial and fungal community composition by non-metric multidimensional scaling (NMDS). The effect of organ and condition on bacterial and fungal community composition was tested with PERMANOVA and 999 random permutations (Adonis tests). Finally, the taxonomic composition of bacterial and fungal communities was compared between conditions by generating heat tree plots and testing differences between the median proportion of reads between conditions using Wilcox rank-sum tests.

\section{Results}

Microbiome diversity and community structure in vanilla. Our metabarcoding data revealed that a total of $1,054,99716 \mathrm{~S}$ reads passed quality filtering and clustered into 1666 bacterial OTUs. The most speciesrich phyla were Proteobacteria (573 OTUs), Actinobacteria (277), Bacteroidetes (245), Acidobacteria (125) and Planctomycetes (124), while 97 OTUs remained unassigned at phylum level. OTU richness was significantly higher in root (1594 OTUs) compared to stem (693) samples when comparing both observed richness $(\chi 2=9.9265, p$-value $=0.002)$ and Shannon index $(\chi 2=8.6471$, p-value $=0.003)$. When looking at species diversity per condition (Table 1), root OTU richness was higher in symptomatic (1385 OTUs) and asymptomatic (1321) compared to feral (763) individuals, while stem OTU richness was higher in feral (598) compared to symptomatic (305) and asymptomatic (163) individuals. These differences however remain non-significant based on pairwise Wilcoxon tests for both observed richness and Shannon index, potentially due to the low number of samples studied. Based on NMDS ordination (Figure 1), the bacterial community composition varied most significantly between organs (R2 $=0.91$, pvalue $=0.001)$, while the effect of condition $(R 2=0.03$, $p$-value $=0.003)$ and the combination of both factors $(R 2=0.06, p$-value $=0.002)$ were lower but nevertheless significant.

Regarding fungi, 1,117,927 ITS2 reads passed quality-filtering and clustered into 889 fungal OTUs belonging to the phyla Ascomycota (607 OTUs), Basidiomycota (121), Mucoromycota (12, sensu Spatafora et al. 2016), Rozellomycota (6), Chytridiomycota (1) and Kickxellomycota (1), while 141 OTUs remained unassigned at phylum level. In addition, 403 OTUs remained unassigned at family level, emphasizing the large number of undescribed fungal groups that are associated with vanilla. The most 
species-rich families were Nectriaceae (32 OTUs), Herpotrichiellaceae (22), Aspergillaceae (14), Cyphellophoraceae (10) and Glomerellaceae (6). Similarly, to what was observed in the bacteria dataset, OTU richness was significantly higher in root (772 OTUs) than in stem (363) samples when comparing observed richness $(\chi 2=8.04, p$-value $=0.005)$, but not Shannon index $(\chi 2=3.19, p$-value $=0.07)$. When looking at species diversity per condition (Table 1), root OTU richness was higher in symptomatic (510 OTUs) than in asymptomatic (438) and wild (303) individuals, while stem OTU richness was higher in feral (226) compared to symptomatic (112) and asymptomatic (98) individuals. Similarly, to what was observed in the bacteria dataset, these differences remain non-significant based on pairwise Wilcoxon tests for both observed richness and Shannon index, potentially due to the low number of samples studied. Fungal community composition varied most significantly between organs $(R 2=0.66, p$-value $=$ $0.001)$, but a good portion of the variation was also explained by condition $(R 2=0.18, p$-value $=0.001)$ and the combination of both factors $(R 2=0.11$, $p$-value $=0.003)$.

Fungal diversity detected from culture isolates. Microbiological isolation of vanilla roots and stem led to 100 fungal isolates growing on PDA, 34 on Sabourad and 10 on Czapek media, for a total of 144 axenic cultures. The isolates were grouped into 58 morphotypes for which we obtained ITS sequences that were deposited in GenBank (MZ270645-MZ270702) (Table S1). Based on ITS sequences we were able to identify 14 genera, predominantly from the phylum Ascomycota, except for two isolates belonging to Basidiomycota. 41 out of 51 morphotypes were identified at family level, distributed in 8 families: Xylariaceae (18 morphotypes), Nectriaceae (7), Stachybotryaceae (5), Biatriosporaceae (4), Bionectriaceae (2), Irpiceae (2), Trichosphaeriaeae (2) and Aspergillaceae (1). In the Xylariaceae ITS phylogenetic tree, our sequences are grouped within the genera Daldinia, Xylaria and Entonaema (Suppl. Fig. S2). When comparing Xylariaceae ITS sequences in UNITE database, the 18 sequences clustered into only four SH identified as Hypoxylon griseobrunneum, Xylaria multiplex, Xylariaceae and Daldinia starbaeckii, each corresponded to an OTU from the metabarcoding analysis. These results indicate that Xylariaceae SH and OTUs may show a high phenotypic diversity of morphotypes when isolated in culture media; and it also suggests that the use of morphotypes is limited in identifying and selecting Xylariaceae. Interestingly, these 4 cultivable OTUs, were present only in asymptomatic samples according to metabarcoding analysis. The second most-species rich family recovered from our cultures was Nectriaceae that includes the genus Fusarium. Nectriaceae ITS sequences from vanilla clustered in the phylogenetic tree with Fusarium solani, Fusarium oxysporum and Fusarium incarnatum-equiseti species complexes (Suppl. Fig. S1). In the UNITE database, these Fusarium sequences were identified as SH corresponding only to Fusarium pseudensiforme and Gibberella fujikuroi. In addition, according to metabarcoding data, Nectriaceae morphotypes sequences matched with 6 different OTUs identified as Fusarium, Nectriaceae and Sordariomycetes in the metabarcoding analysis. The OTUs matching these isolates were present predominantly in the root samples (Table S1).

Most abundant taxa in vanilla microbiome. Even though the frequency of bacterial and fungal phyla is similar between conditions in both root and stem samples (Figure 2), when analyzing the 10 most frequent bacterial and fungal families in our dataset, marked differences in OTU frequencies were observed between both condition asymptomatic, cultivated/symptomatic, cultivated/feral 
(asymptomatic, symptomatic, and feral) and organ type. Unfortunately, because of the very small number of feral samples, we could not analyze differences between feral and cultivated (as two states). Asymptomatic stems shared the bacterial family Sphingomonadaceae, and the fungal families Phaeosphaeriaceae, Orbiliaceae and Glomerellaceae, while Pseudonocardiaceae (bacteria) and Nectriaceae (fungi) were almost absent. The relative abundance among bacterial taxa (Figure 3 ) shows that the family Beijerinckaceae as well as various Actinobacteria were significantly more abundant in feral individuals, while Proteobacteria were significantly more abundant in asymptomatic individuals. The class Negativicutes was also significantly more abundant in asymptomatic individuals than in symptomatic individuals. Commonly isolated bacterial genera included Paenibacillus from orchid meristems [38] and Streptomyces, Bacillus, Flavobacterium, and Pseudomonas from orchid roots [39,40], which we recovered in almost all our samples. Regarding fungi, the families Mycosphaerellaceae, Neodevriesiaceae Sporocadaceae, Stachybotryaceae, Teratosphaeriaceae, Tremellaceae, Xylariaceae, as well as the order Chaetothryriales were significantly more abundant in symptomatic individuals (Fig. 3). Mycosphaerellaceae was also significantly more abundant in feral than asymptomatic individuals, while Neodevriesiaceae was less abundant in feral plants than cultivated symptomatic individuals. Although the family Nectrinaceae (that comprise the genus Fusarium) was more abundant in asymptomatic than symptomatic cultivated plants and feral individuals (Figure S3), these differences however stayed nonsignificant according to Wilcoxon Rank Sum tests. Porras-Alfaro and Bayman (2007) previously reported Tulasnella, Ceratobasidium y Thanatephorus from microbiological isolates characterized by ITS Vanilla planifolia y poitaei. We recovered Ceratobasidium in our microbiological isolates in our live collection, and in the metabarcoding data.

\section{Discussion}

The taxonomic composition of the endophytic microbiome of Mexican native Vanilla planifolia was characterized in plants affected by stem and root rot disease, with the hypothesis that the presence of Fusarium oxysporum $f$. sp. vanillae alone, is not enough to explain symptoms, and that the diversity of the microbiome matters. The anthropogenic effects of long-term cultivation in its microbiomes have not been studied enough in $V$. planifolia to identify overall trends. We found that Proteobacteria and Actinobacteria, as well as the fungal families Nectriaceae and Xylariaceae constitute the core of the vanilla microbiome in the root and stem of both cultivated and feral plants. Cultivated taxa from our microbiological collection characterized with Sanger sequencing also mostly belong to the Xylariaceae and Nectriaceae families, although it is important to note that species remain hypothetical due to the difficulties in taxonomic assignment in fungi. Ceratobasidium seems to be an important genus in vanilla as previously identified for this species [41], although its function is not clear, whether it is endomycorrhizal or saprotrophic, for instance. Members of the Xylariaceae in general likely have an important role in the expression of symptoms of SRD. The life cycle of members of this family usually starts with an endophytic phase where the spores and mycelia live inside plant tissues without causing any visible symptoms. If the plant undergoes abiotic or biotic stress, members of the Xylariales become 
saprotrophic fungi that feed from previously infected tissue, contributing to a faster rotting of the affected area [42].

We did not detect significant differences in bacterial or fungal overall levels of diversity among cultivated vanilla and its feral counterpart, or among asymptomatic and symptomatic individuals. This is similar to a recent report in vanilla plants growing in wild-natural conditions in which the community of mycorrhizal fungi was not significantly different to the plants in the highly managed farm [41]. However, diversity indexes and richness comparisons can be sensitive to our small sample size, so we focused on the 10 most abundant taxa, for which we found differences in groups such as Xylariaceae and Actinobacteria and Proteobacteria, among conditions and organs, as well as some more unusual groups such as Negativicutes, apparently present only in asymptomatic individuals. Also, while not significant, there was generally lower diversity in asymptomatic plants, independent of methodology, perhaps due to pathogen competition in the plant. A lower diversity of endophytic microbiomes in infected/symptomatic plants when compared to healthy samples was observed in apple flowers after Erwinia amylovora phytopathogen inoculation in contrast to water inoculated flowers [43]. Richness of endophytic fungal morphotypes in bananas cv. Manzano was higher in healthy plants compared with diseased plants with Fusarium oxysporum f. sp. cubense according to microbiological endophytic isolation [44].

Interestingly, we did find significant differences in the richness and diversity of the Shannon index between organs, stem and root, with the root being most diverse for both bacteria and fungi. This suggests host-driven specificity for each organ, similar to three Mediterranean terrestrial orchids, Neottia ovata, Serapias vomeracea, and Spiranthes spiralis, whose organ-specific microbiome appears to have an active involvement in the orchid holobiont [45].

The species composition patterns that we found do not suggest any clear evidence that Fusarium is the causing agent of the SRD symptoms, confirming our main hypothesis that SRD is the expression of additional factors other than Fusarium alone. This is also congruent with studies that have hypothesized that Fusarium spp can prevent other fungi from establishing and growing through direct competition, hindering the growth of the rest of the community by modifying the environment within the tissue in their favor $[6,11,12]$. This effect has also been reported in wheat (Triticum aestivum) ears [46] and a decrease in fungal endophyte diversity has been observed in rot diseases caused by Fusarium spp. (mainly $F$. graminearum and F. culmorum). The Nectrinaceae family that includes Fusarium species was generally more abundant in asymptomatic than symptomatic and feral individuals, supporting a more complex ecological and biological role for Fusarium, but our small sample size limited the statistical power to confirm this trend. Fusarium and other members of Nectriaceae are usually predominant in the root compared to the stem. The root is thought to be the point of entry for Fusarium [15] where we expect the highest fungal diversity to occur naturally, and where their ecological role may be broader. Furthermore, our phylogenetic analyses suggest that the Fusarium present in Mexican vanilla is a species complex, rather than a single species that could be associated to SRD, and that the pathogenic nature of Fusarium almost certainly depends on other factors such as previously described pathogenic genomic regions for other plants $[47,48]$. Fusarium species, historically associated with SRD, are likely present in 
the plant in both cultivated and feral conditions as part of the extended microbiome, and the expression of symptoms is associated with additional factors, rather than with its simple presence or absence.

\section{Declarations}

Acknowledgments - Funding for this project was provided to ACJ by a UCMEXUS grant and FBG by Conacyt, Mexico (grant No. 285746) and the Royal Society of the United Kingdom, Newton Advanced Fellowship (NAF\R2\180631).

Conflicts of interest/Competing interests - No conflicts of interest or competing interests.

Authors' contributions (optional: please review the submission guidelines from the journal whether statements are mandatory) - ICV and AHMS completed the field work, extracted DNA, generated the microbiological live collection, and analyzed the data. CT analyzed the data and contributed significantly to the revision of the manuscript, as well as conceptually to the methods. JHH and RD assisted in the field and are the owners of live vanilla collections or feral plants where all the samples were obtained for this study. FBG assisted in the field and contributed to the microbiological live collection, as well as provided partial funding. ACJ conceived the study, provided funding, led sample collections in the field and wrote most of the manuscript.

Availability of data and material (data transparency) - The nucleotide sequence data reported are available in the DDBJ/EMBL/GenBank databases under the accession number(s) as follows: 
Isolate ID Taxonomical classification

GB

Accession

VPI_S15 Fungi; Dikarya; Ascomycota; Pezizomycotina; Sordariomycetes;

MZ270645

Xylariomycetidae; Xylariales; Xylariaceae; Daldinia

VPI_A20 Fungi; Dikarya; Ascomycota; Pezizomycotina; Sordariomycetes;

Xylariomycetidae; Xylariales; Xylariaceae; Daldinia

MZ270646

VPI_S31 Fungi; Dikarya; Ascomycota; Pezizomycotina; Sordariomycetes;

Xylariomycetidae; Xylariales; Xylariaceae; Daldinia

MZ270647

VPI_S63 Fungi; Dikarya; Basidiomycota; Agaricomycotina; Agaricomycetes;

Agaricomycetes sbc Incertae sedis; Polyporales; Irpicaceae; Ceriporia

MZ270648

VPI_S16 Fungi; Dikarya; Basidiomycota; Agaricomycotina; Agaricomycetes;

Agaricomycetes sbc Incertae sedis; Polyporales; Irpicaceae

MZ270649

VPI_S35 Fungi; Dikarya; Ascomycota; Pezizomycotina; Sordariomycetes;

Hypocreomycetidae; Hypocreales; Bionectriaceae; Clonostachys

MZ270650

VPI_S51 Fungi; Dikarya; Ascomycota; Pezizomycotina; Sordariomycetes;

Hypocreomycetidae; Hypocreales; Bionectriaceae; Clonostachys

MZ270651

VPI_S1 Fungi; Dikarya; Ascomycota; Pezizomycotina; Eurotiomycetes;

Eurotiomycetidae; Eurotiales; Aspergillaceae; Penicillium

MZ270652

VPI_A12 Fungi; Dikarya; Ascomycota

MZ270653

VPI_S18b Fungi; Dikarya; Ascomycota

MZ270654

VPI_A29 Fungi; Dikarya; Ascomycota

MZ270655

VPI_S65 Fungi; Dikarya; Ascomycota

MZ270656

VPI_S13 Fungi; Dikarya; Ascomycota; Pezizomycotina; Sordariomycetes;

Xylariomycetidae; Xylariales

MZ270657

VPI_S19 Fungi; Dikarya; Ascomycota; Pezizomycotina; Sordariomycetes;

Xylariomycetidae; Xylariales

MZ270658

VPIS21 Fungi; Dikarya; Ascomycota; Pezizomycotina; Sordariomycetes;

Xylariomycetidae; Xylariales

MZ270659

VPI_S28 Fungi; Dikarya; Ascomycota; Pezizomycotina; Sordariomycetes;

Xylariomycetidae; Xylariales

MZ270660

VPI_S41 Fungi; Dikarya; Ascomycota; Pezizomycotina; Sordariomycetes;

Xylariomycetidae; Xylariales

MZ270661

VPI_S42 Fungi; Dikarya; Ascomycota; Pezizomycotina; Sordariomycetes; Xylariomycetidae; Xylariales

MZ270662

VPI_S46 Fungi; Dikarya; Ascomycota; Pezizomycotina; Sordariomycetes; Xylariomycetidae; Xylariales

MZ270663

VPI_A47 Fungi; Dikarya; Ascomycota; Pezizomycotina; Sordariomycetes; Xylariomycetidae; Xylariales 
VPI_S52 Fungi; Dikarya; Ascomycota; Pezizomycotina; Sordariomycetes;

Xylariomycetidae; Xylariales

MZ270665

VPI_S5 Fungi; Dikarya; Ascomycota; Pezizomycotina; Sordariomycetes;

Xylariomycetidae; Xylariales

MZ270666

VPI_S24 Fungi; Dikarya; Ascomycota; Pezizomycotina; Sordariomycetes;

MZ270667

Xylariomycetidae; Xylariales; Xylariaceae; Xylaria

VPI_S30 Fungi; Dikarya; Ascomycota; Pezizomycotina; Sordariomycetes;

Xylariomycetidae; Xylariales; Xylariaceae; Xylaria

MZ270668

VPI_S32 Fungi; Dikarya; Ascomycota; Pezizomycotina; Sordariomycetes;

Xylariomycetidae; Xylariales; Xylariaceae; Xylaria

MZ270669

VPI_S54 Fungi; Dikarya; Ascomycota; Pezizomycotina; Sordariomycetes;

MZ270670

Xylariomycetidae; Xylariales; Xylariaceae; Xylaria

VPI_A11 Fungi; Dikarya; Ascomycota; Pezizomycotina; Sordariomycetes;

Xylariomycetidae; Xylariales; Xylariaceae; Hypoxylon

MZ270671

VPI_S14 Fungi; Dikarya; Ascomycota; Pezizomycotina; Sordariomycetes;

Xylariomycetidae; Xylariales; Xylariaceae; Hypoxylon

MZ270672

VPI_S17b Fungi; Dikarya; Ascomycota; Pezizomycotina; Sordariomycetes;

MZ270673

Hypocreomycetidae; Hypocreales; Nectriaceae; Fusarium

VPI_A22 Fungi; Dikarya; Ascomycota; Pezizomycotina; Sordariomycetes;

Hypocreomycetidae; Hypocreales; Nectriaceae; Fusarium

MZ270674

VPI_S45 Fungi; Dikarya; Ascomycota

MZ270675

VPI_A39 Fungi; Dikarya; Ascomycota; Pezizomycotina; Sordariomycetes;

MZ270676

Sordariomycetes sbc Incertae sedis; Trichosphaeriales;

Trichosphaeriaceae; Nigrospora

VPI_A3 Fungi; Dikarya; Ascomycota; Pezizomycotina; Sordariomycetes;

Sordariomycetes sbc Incertae sedis; Trichosphaeriales;

MZ270677

Trichosphaeriaceae; Nigrospora

VPI_S10 Fungi; Dikarya; Ascomycota; Pezizomycotina; Sordariomycetes;

Hypocreomycetidae; Hypocreales; Stachybotryaceae; Myxospora

MZ270678

VPI_S17 Fungi; Dikarya; Ascomycota; Pezizomycotina; Sordariomycetes;

Hypocreomycetidae; Hypocreales; Stachybotryaceae; Myxospora

MZ270679

VPI_S38 Fungi; Dikarya; Ascomycota; Pezizomycotina; Sordariomycetes;

Hypocreomycetidae; Hypocreales; Stachybotryaceae; Myxospora

MZ270680

VPI_S66 Fungi; Dikarya; Ascomycota; Pezizomycotina; Sordariomycetes;

MZ270681

Hypocreomycetidae; Hypocreales; Stachybotryaceae; Myxospora

VPI_A25 Fungi; Dikarya; Ascomycota; Pezizomycotina; Sordariomycetes;

Hypocreomycetidae; Hypocreales; Stachybotryaceae; Myxospora

MZ270682

VPI_S64 Fungi; Dikarya; Ascomycota; Pezizomycotina; Dothideomycetes;

Pleosporomycetidae

MZ270683

VPI_S70 Fungi; Dikarya; Ascomycota

MZ270684 
VPI_S72 Fungi; Dikarya; Ascomycota

MZ270685

VPI_A73 Fungi; Dikarya; Ascomycota

MZ270686

VPI_S8 Fungi; Dikarya; Ascomycota

MZ270687

VPI_A1a Fungi; Dikarya; Ascomycota; Pezizomycotina; Sordariomycetes;

MZ270702

Hypocreomycetidae; Hypocreales; Nectriaceae; Gibberella

VPI_S1b Fungi; Dikarya; Ascomycota; Pezizomycotina; Sordariomycetes;

Hypocreomycetidae; Hypocreales; Nectriaceae; Gibberella

MZ270688

VPI_A23 Fungi; Dikarya; Ascomycota; Pezizomycotina; Sordariomycetes;

Hypocreomycetidae; Hypocreales; Nectriaceae; Gibberella

MZ270689

VPI_A27 Fungi; Dikarya; Ascomycota; Pezizomycotina; Sordariomycetes;

MZ270690

Hypocreomycetidae; Hypocreales; Nectriaceae; Gibberella

VPI_S2a Fungi; Dikarya; Ascomycota; Pezizomycotina; Sordariomycetes;

MZ270691 Hypocreomycetidae; Hypocreales; Nectriaceae; Gibberella

VPI_S62 Fungi; Dikarya; Ascomycota; Pezizomycotina; Sordariomycetes; Hypocreomycetidae; Hypocreales

MZ270692

VPI_S7 Fungi; Dikarya; Ascomycota; Pezizomycotina; Sordariomycetes; Hypocreomycetidae; Hypocreales

MZ270693

VPI_A9 Fungi; Dikarya; Ascomycota; Pezizomycotina; Sordariomycetes; Hypocreomycetidae; Hypocreales

MZ270694

VPI_S26 Fungi; Dikarya; Ascomycota; Pezizomycotina; Sordariomycetes;

MZ270695 Hypocreomycetidae

VPI_S33 Fungi; Dikarya; Ascomycota; Pezizomycotina

MZ270696

VPI_A34 Fungi; Dikarya; Ascomycota; Pezizomycotina; Dothideomycetes; Pleosporomycetidae; Pleosporales; Biatriosporaceae; Biatriospora

MZ270697

VPI_S36 Fungi; Dikarya; Ascomycota; Pezizomycotina; Dothideomycetes; Pleosporomycetidae; Pleosporales; Biatriosporaceae; Biatriospora

MZ270698

VPI_S53 Fungi; Dikarya; Ascomycota; Pezizomycotina; Dothideomycetes;

Pleosporomycetidae; Pleosporales; Biatriosporaceae; Biatriospora

MZ270699

VPI_A55 Fungi; Dikarya; Ascomycota; Pezizomycotina; Dothideomycetes;

Pleosporomycetidae; Pleosporales; Biatriosporaceae; Biatriospora

MZ270700

VPI_A59 Fungi; Dikarya; Ascomycota; Pezizomycotina; Dothideomycetes; Pleosporomycetidae; Pleosporales; Pleosporales fam Incertae sedis;

MZ270701 Nigrograna

Code availability (software application or custom code) - In-house scripts are available: Muñoz-Sanchez AH, Troung C Microbial diversity in Vanilla planifolia orchids using metabarcoding analysis.

https://github.com/arihelrnan/Endophyte_microbiome_Vanilla_planifolia 
Ethics approval (include appropriate approvals or waivers) - Not applicable

Consent to participate (include appropriate statements) - Not applicable

Consent for publication (include appropriate statements)- Not applicable

Additional declarations for articles in life science journals that report the results of studies involving humans and/or animals - Not applicable

\section{References}

1. Espinoza-Pérez, J (2016) Estudio de factores socio-culturales que influyen en el manejo de la vainilla (Vanilla planifolia Jacks. ex Andrews), en la región Totonacapan, México. Dissertation. Colegio de Postgraduados.

2. Food and Agriculture Organization Data (FAOSTAT). 2017.

http://faostat3.fao.org/download/Q/QC/S (23 marzo de 2017).

3. Pinaria AG, Liew ECY, Burgess LW (2010) Fusarium species associated with vanilla stem rot in Indonesia. Australas. Plant Pathol. https://doi.org/10.1071/AP090796.

4. Tucker GM (1927) Vanilla Root Rot. J Agric Res 35:1121-36.

5. Hernández, J (2011) Programa Estratégico para el desarrollo rural sustentable de la Región sur-este de México Trópico húmedo 2011. Paquete Tecnológico Vainilla (Vanilla planifolia Jackson) Establecimiento y mantenimiento. Inifap (Instituto Nacional de Investigaciones Forestales, Agricolas y Pecuarias).

6. Hernández-Martínez JL, Carranza-Álvarez C, Maldonado-Miranda JJ, Martínez-Soto D. Isolation of Fusarium from vanilla plants grown in the Huasteca Potosina Mexico. Rev Mex Fitopatol Mex J Phytopathol. 2020;38(3):475-84.

7. Adame-García J, Rodríguez-Guerra R, Iglesias-Andreu LG, Ramos-Prado JM, Luna-Rodríguez M (2015) Molecular Identification and Pathogenic Variation of Fusarium species isolated from Vanilla planifolia in Papantla Mexico. Botanical Sciences 93: 669-678. https://doi.org/10.17129/botsci.142

8. Koyyappurath S, Conéjéro G, Dijoux JB, Lapeyre-Montès F, Jade K, Chiroleu F, Gatineau F, Verdeil JL, Besse P, Grisoni M (2015) Differential Responses of Vanilla Accessions to Root Rot and Colonization by Fusarium oxysporum $f$. sp. radicis-vanillae. Frontiers in Plant Science 6:1-16. https://doi.org/10.3389/fpls.2015.01125

9. Koyyappurath S, Atuahiva T, Le Guen R, Batina H, Le Squin S, Gautheron N, Edel HV, Peribe J, Jahiel M, Steinberg C, Liew ECY, Alabouvette C, Besse P, Dron M, Sache I, Laval V, Grisoni M (2016) Fusarium oxysporum $f$. sp. radicis-vanillae is the causal agent of root and stem rot of vanilla. Plant Pathology 65:612-625. https://doi.org/10.1111/ppa.12445

10. Cardona C S, Díez MC (2012) Identificación del agente causal de la pudrición basal del tallo de vainilla en cultivos bajo cobertizos en Colombia. Revista Mexicana de Micología 35:23-34. 
11. Gamboa-Gaitán M (2013). Colombian Vanilla and its microbiota, I First report of Fusarium taxa from wild and cultivated species. Acta Botanica Hungarica 55:429-472.

https://doi.org/10.1556/ABot.55.2013.3

12. Khoyratty S, Dupont J, Lacoste S, Palama LT, Choi HY, Kim KH, Payet B, Grisoni M, Fouillaud M, Verpoorte R, Kodja H (2015) Fungal endophytes of Vanilla planifolia across Réunion Island: Isolation, distribution and biotransformation. BMC Plant Biology 15:1-19. https://doi.org/10.1186/s12870015-0522-5

13. Nel B, Steinberg C, Labuschagne N, Viljoen A (2006) The potential of nonpathogenic Fusarium oxysporum and other biological control organisms for suppressing fusarium wilt of banana. Plant Pathology 55: 217-223. https://doi.org/10.1111/j.1365-3059.2006.01344.x

14. White JF Jr, Torres MS, Sullivan RF, Jabbour RE, Chen Q, Tadych M, Irizarry I, Bergen MS, HavkinFrenkel D, Belanger FC (2014) Occurrence of Bacillus amyloliquefaciens as a systemic endophyte of vanilla orchids. Microscopy Research and Technique 77:874-885. https://doi.org/10.1002/jemt.22410

15. Xiong W, Li R, Ren Y, Liu C, Zhao Q, Wu H, Jousset A, Shen Q (2017) Distinct roles for soil fungal and bacterial communities associated with the suppression of vanilla Fusarium wilt disease. Soil Biology and Biochemistry 107:198-207. https://doi.org/10.1016/j.soilbio.2017.01.010

16. Xiong W, Guo S, Jousset A, Zhao Q, Wu H, Rong L, Kowalchuk G, Shen Q (2017) Bio-fertilizer application induces soil suppressiveness against Fusarium wilt disease by reshaping the soil microbiome. Soil biology and biochemistry 114:238-247.

https://doi.org/10.1016/j.soilbio.2017.07.016

17. Cenis JL (1992) Rapid extraction of fungal DNA for PCR amplification. Nucleic Acids Res 20:2380. https://doi.org/10.1093/nar/20.9.2380

18. Kim JS, Seo SG, Jun BK, Kim JW, Kim SH (2010) Simple and reliable DNA extraction method for the dark pigmented fungus, Cercospora sojina. Plant Pathol JI 26: 289-292. https://doi.org/10.5423/PPJ.2010.26.3.289

19. Gardes M, Bruns TD (1993) ITS primers with enhanced specificity for basidiomycetes - application to the identification of mycorrhizae and rusts. Mol Ecol 2: 113-118. ttps://doi.org/10.1111/j.1365294X.1993.tb00005.x

20. White TJ, Bruns T, Lee S, Taylor J (1990) Amplification and direct sequencing of fungal ribosomal RNA genes for phylogenetics. In PCR Protocols: A guide to methods and applications 55:315-322.

21. Nilsson RH, Larsson KH, Taylor AFS, Bengtsson-Palme J, Jeppesen TS, Schigel D, Kennedy P, Picard K, Glöckner FO, Tedersoo L, Saar I, Kõljalg, U, Abarenkov K (2019). The UNITE database for molecular identification of fungi: Handling dark taxa and parallel taxonomic classifications. Nucleic Acids Res, 47: D259-D264. https://doi.org/10.1093/nar/gky1022

22. Edgar RC (2004) MUSCLE: Multiple sequence alignment with high accuracy and high throughput. Nucleic Acids Res 32: 1792-1797. https://doi.org/10.1093/nar/gkh340 
23. Castresana $J$ (2000) Selection of conserved blocks from multiple alignments for their use in phylogenetic analysis. Mol. Biol. Evol. 17: 540-552.

https://doi.org/10.1093/oxfordjournals.molbev.a026334

24. Kumar S, Stecher G, Li M, Knyaz C, Tamura K (2018) MEGA X: Molecular evolutionary genetics analysis across computing platforms. Mol. Biol. Evol. 35: 1547-1549.

https://doi.org/10.1093/molbev/msy096

25. Japelaghi RH, Haddad R, Garoosi GA (2011) Rapid and Efficient Isolation of High Quality Nucleic Acids from Plant Tissues Rich in Polyphenols and Polysaccharides. Mol Biotechnol 49:129-137. https://doi.org/10.1007/s12033-011-9384-8

26. Inglis PW, Pappas MdCR, Resende LV, Grattapaglia D (2018) Fast and inexpensive protocols for consistent extraction of high quality DNA and RNA from challenging plant and fungal samples for high-throughput SNP genotyping and sequencing applications. PLoS ONE 13: e0206085. https://doi.org/10.1371/journal.pone.0206085

27. Klindworth A, Pruesse E, Schweer T, Peplies J, Quast C, Horn M, Glöckner OF (2013) Evaluation of general $16 \mathrm{~S}$ ribosomal RNA gene PCR primers for classical and next-generation sequencing-based diversity studies. Nucleic Acids Res 41:e1 https://doi.org/10.1093/nar/gks808

28. Palmer JM, Jusino MA, Banik MT, Lindner DL (2018) Non-biological synthetic spike-in controls and the AMPtk software pipeline improve mycobiome data. PeerJ 6:e4925; DOI 10.7717/peerj. 4925.

29. Cole JR, Wang Q, Fish JA, Chai B, McGarrell DM, Sun Y, Brown CT, Porras-Alfaro A, Kuske CR, Tiedje JM (2014) Ribosomal Database Project: data and tools for high throughput rRNA analysis. Nucleic Acids Res 42:D633-D642, https://doi.org/10.1093/nar/gkt1244

30. Caporaso JG, Kuczynski J, Stombaugh J, Bittinger K, Bushman FD, Costello EK, Fierer N, Pena AG, Goodrich JK, Gordon JI, Huttley GA, Kelley ST, Knights D, Koenig JE, Ley RE, Lozupone CA, McDonald D, Muegge BD, Pirrung M, Reeder J, Sevinsky JR, Turnbaugh PJ, Walters WA, Widmann J, Yatsunenko T, Zaneveld J, Knight R (2010) QIIME allows analysis of highthroughput community sequencing data. Nat. Methods 7(5):335-336 doi:10.1038/nmeth.f.303

31. McMurdie PJ, Holmes S (2013) phyloseq: An R package for reproducible interactive analysis and graphics of microbiome census data. PLoS ONE 8(4): e61217.

http://dx.plos.org/10.1371/journal.pone.0061217.

32. Wickham H (2016) ggplot2: Elegant Graphics for Data Analysis Springer-Verlag New York. ISBN 9783-319-24277-4. https://ggplot2.tidyverse.org.

33. Foster ZSL, Sharpton TJ, Grünwald NJ (2017) Metacoder: An R package for visualization and manipulation of community taxonomic diversity data. PLOS Comput Biol. 13(2): e1005404. https://doi.org/10.1371/journal.pcbi.1005404

34. Oksanen J, Blanchet FG, Friendly M, Kindt R, Legendre P, McGlinn D, Minchin PR, O'Hara RB, Simpson GL, Solymos P, Stevens MHH, Szoecs E, Wagner H (2019) vegan: Community Ecology Package. R package version 2.5-6. https://CRAN.R-project.org/package=vegan 
35. Fernandez CW, Nguyen NH, Stefanski A, Han Y, Hobbie SE, Montgomery RA, Reich PB, Kennedy PG (2017) Ectomycorrhizal fungal response to warming is linked to poor host performance at the borealtemperate ecotone. Glob. Change Biol. 23: 1598-1609. DOI: 10.1111/gcb.13510

36. Nguyen NH, Smith D, Peay K, Kennedy P (2015) Parsing ecological signal from noise in next generation amplicon sequencing. New Phytol. 205: 1389- 1393. https://doi.org/10.1111/nph.12923

37. Pickles BJ, Truong C, Watts-Williams SJ, Bueno CG (2020) Mycorrhizas for a sustainable world. New Phytol. 225: 1065-1069. https://doi.org/10.1111/nph.16307

38. Faria D C, Dias ACF, Melo IS, de Carvalho Costa FE (2013). Endophytic bacteria isolated from orchid and their potential to promote plant growth. World J Microbiol Biotechnol 29:217-221 https://doi.org/10.1007/s11274-012-1173-4

39. Tsavkelova EA, Cherdyntseva TA, Netrusov Al (2004). Bacteria associated with the roots of epiphytic orchids. Microbiology 73: 710-715. doi: 10.1007/s11021-005-0013-z

40. Wilkinson KG, Dixon KW, Sivasithamparam K, Ghisalberti E L (1994). Effect of IAA on symbiotic germination of an Australian orchid and its production by orchid-associated bacteria. Plant Soil 159, 291-295. doi: 10. 1007/BF00009292

41. Johnson LJAN, González-Chávez MdC, Carrillo-González R, Porras-Alfaro A, Mueller GM (2020) Vanilla Aerial and Terrestrial Roots Host Rich Communities of Orchid Mycorrhizal and Ectomycorrhizal Fungi. Plants, People, Planet, no. November 2019: 1-12. https://doi.org/10.1002/ppp3.10171.

42. Whalley AJS (1996) The xylariaceous way of life. Mycol Res 100(8):897-922. doi: http://dx.doi.org/10.1016/S0953-7562(96)80042-6

43. Cui Z, Huntley RB, Zeng Q, Steven B (2021) Temporal and spatial dynamics in the apple flower microbiome in the presence of the phytopathogen Erwinia amylovora. ISME Journal, 15: 318-329. https://doi.org/10.1038/s41396-020-00784-y

44. Zapata-Henao S, Henao-Vasquez MC, Patino-Hoyos LF, Sanchez-Torres JD, Hoyos-Carvajal L M (2019) Fungal endophytes in bananas cv Manzano affected by Fusarium. African Journal of Agricultural Research, 14(7), 430-438. https://doi.org/10.5897/ajar2018.13736

45. Alibrandi P, Schnell S, Perotto S, Cardinale M (2020) Diversity and Structure of the Endophytic Bacterial Communities Associated With Three Terrestrial Orchid Species as Revealed by 16S RRNA Gene Metabarcoding. Frontiers in Microbiology 11: 1-18. https://doi.org/10.3389/fmicb.2020.604964.

46. Rojas EC, Sapkota R, Jensen B, Jørgensen HJL, Henriksson T, Jørgensen LN, Nicolaisen M, Collinge DB (2019) Fusarium Head Blight Modifies Fungal Endophytic Communities During Infection of Wheat Spikes. Microbial Ecology. https://doi.org/10.1007/s00248-019-01426-3

47. Ma LJ, Van Der Does HC, Borkovich KA, Coleman JJ, Daboussi MJ, Di Pietro A, Dufresne M, Freitag M, Grabherr M, Henrissat B, Houterman P M, Kang S, Shim W B, Woloshuk C, Xie X, Xu JR, Antoniw J, Baker SE, Bluhm BH, ... Rep M (2010) Comparative genomics reveals mobile pathogenicity chromosomes in Fusarium. Nature, 464 367-373. https://doi.org/10.1038/nature08850 
48. van DaM P, Fokkens L, Schmidt SM, Linmans JHJ, Corby Kistler H, Ma LJ, Rep M (2016) Effector profiles distinguish formae speciales of Fusarium oxysporum. Environmental Microbiology, 18(11), 4087-4102. https://doi.org/10.1111/1462-2920.134455

\section{Tables}

Table 1. Mean and standard deviation for alpha diversity values per organ and condition

\begin{tabular}{|c|c|c|c|c|c|c|}
\hline & ROOT & & & STEM & & \\
\hline & Symptomatic & Asymptomatic & Feral & Symptomatic & Asymptomatic & Feral \\
\hline $\begin{array}{l}\text { BACTERIA } \\
\text { observed } \\
\text { richness }\end{array}$ & $1009 \pm 89.72$ & $915 \pm 38$ & $\begin{array}{l}494.50 \\
\pm \\
236.88\end{array}$ & $134 \pm 110.91$ & $66.33 \pm 47.44$ & $\begin{array}{l}442 \pm \\
73.54\end{array}$ \\
\hline $\begin{array}{l}\text { BACTERIA } \\
\text { Shannon } \\
\text { index }\end{array}$ & $4.72 \pm 0.85$ & $4.38 \pm 0.17$ & $\begin{array}{l}3.17 \pm \\
0.68\end{array}$ & $0.85 \pm 0.72$ & $0.57 \pm 0.67$ & $\begin{array}{l}3.33 \\
\pm \\
0.62\end{array}$ \\
\hline $\begin{array}{l}\text { FUNGI } \\
\text { observed } \\
\text { richness }\end{array}$ & $\begin{array}{l}292.33 \pm \\
92.18\end{array}$ & $\begin{array}{l}187.66 \pm \\
167.70\end{array}$ & $\begin{array}{l}178 \pm \\
82.02\end{array}$ & $\begin{array}{l}48.67 \pm \\
30.92\end{array}$ & $35.33 \pm 33.50$ & $\begin{array}{l}130 \pm \\
11.72\end{array}$ \\
\hline $\begin{array}{l}\text { FUNGI } \\
\text { Shannon } \\
\text { index }\end{array}$ & $3.10 \pm 0.76$ & $2.26 \pm 1.28$ & $\begin{array}{l}3.19 \pm \\
0.61\end{array}$ & $2.17 \pm 1.09$ & $1.26 \pm 1.11$ & $\begin{array}{l}2.22 \\
\pm \\
1.06\end{array}$ \\
\hline
\end{tabular}

\section{Figures}
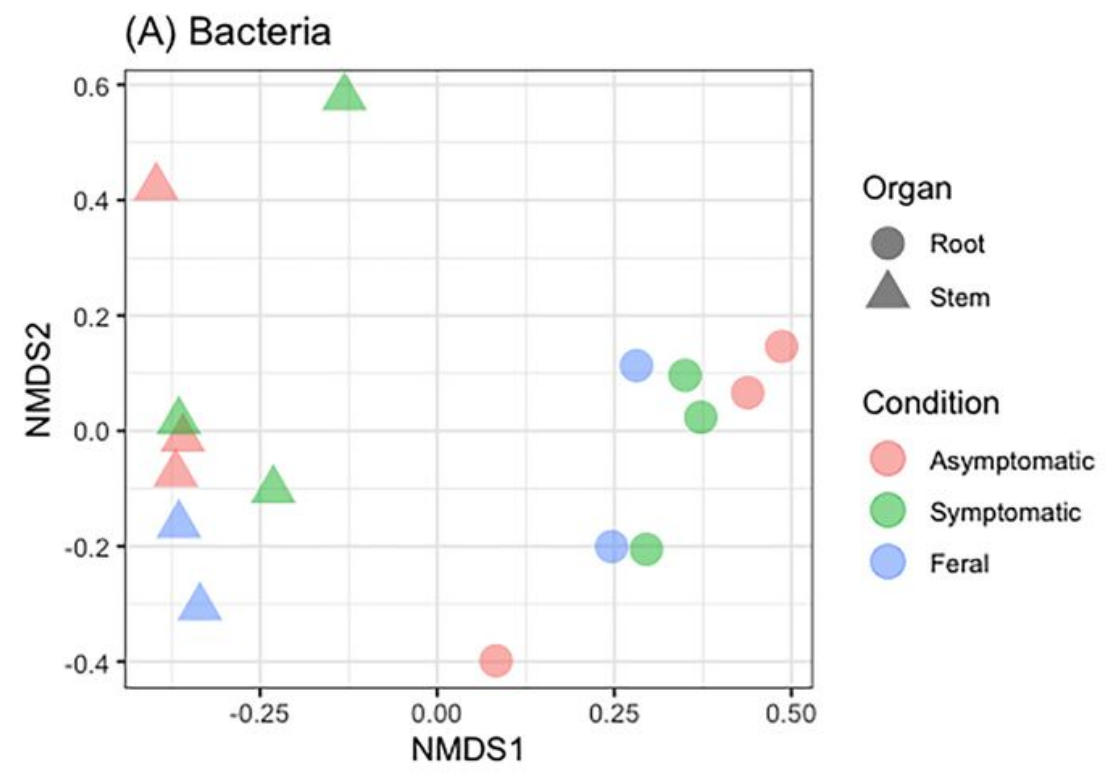

Figure 1

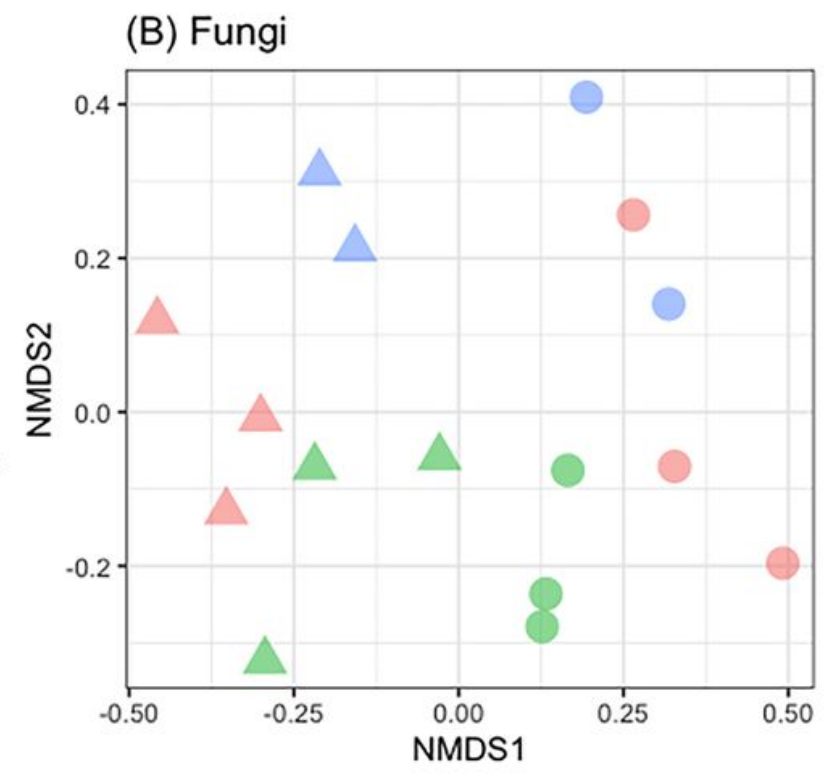


Non-metric multidimensional scaling (NMDS) of (A) bacterial, and (B) fungal community composition based on Raup-Crick distances, with samples shaped by organ (root=circle, stems=triangle) and colored by condition (asymptomatic=red, symptomatic=green, feral =blue)


Figure 2

OTU frequency (A) per bacterial phylum, (B) in the 10 most frequent bacterial families, (C) per fungal phylum and (D) in the 10 most frequent fungal families detected by 16S and ITS2 meta-barcoding of root and stem samples of asymptomatic (Asym), symptomatic (Sym), and feral (Fer) Vanilla individuals 


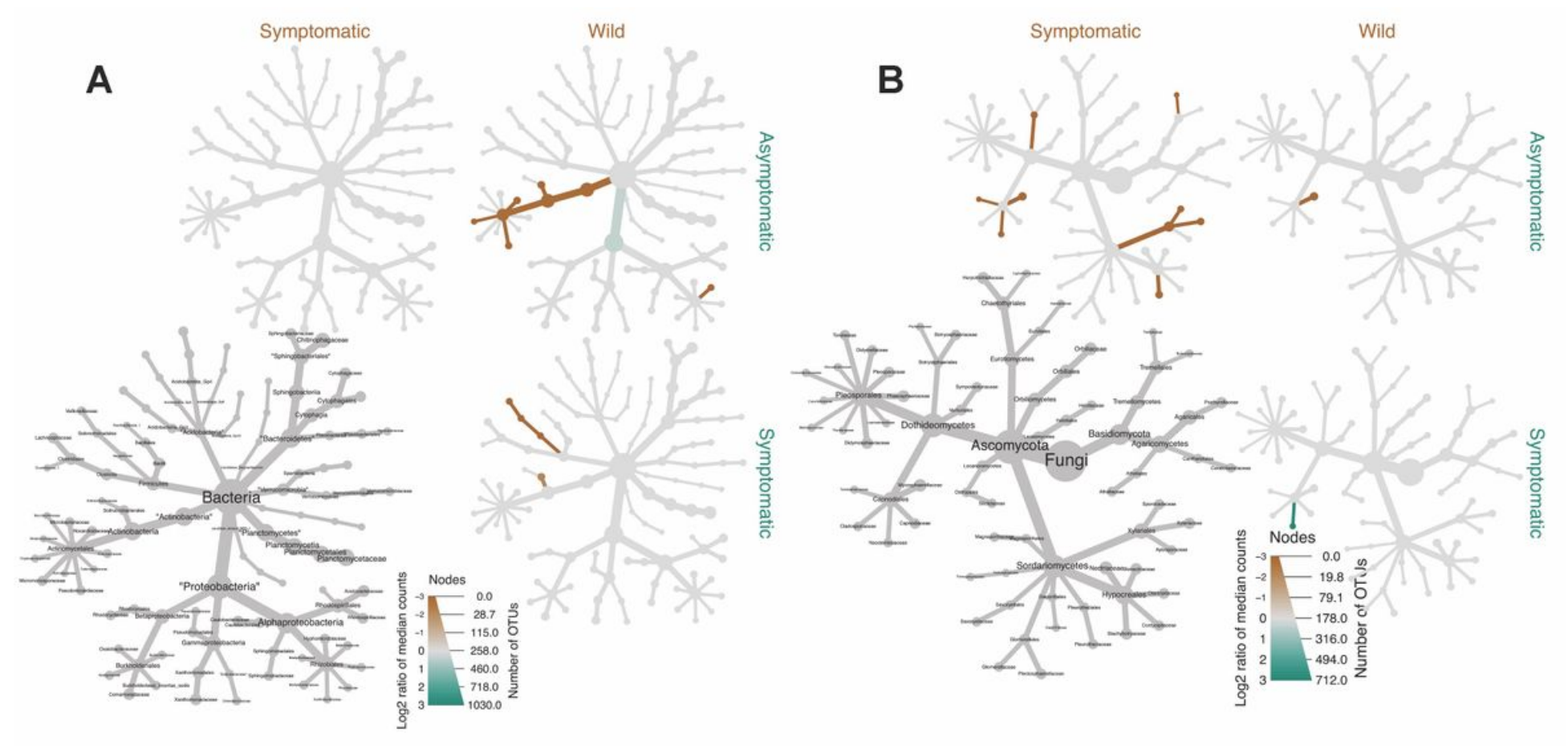

Figure 3

Differential heat tree matrices illustrate significant change in $(A)$ bacterial and $(B)$ fungal relative abundances between conditions at the family level, for taxa with relative abundance $>0.01 \%$ of total read count per sample. The size of nodes in the gray cladograms depicts the number of taxa identified at each taxonomic level. The smaller cladograms represent pairwise comparisons between conditions: an orange node indicates a significantly higher abundance in the condition stated horizontally, according to Wilcoxon Rank Sum tests with FDR correction for multiple comparisons, while a blue-green node indicates a significantly higher abundance in the condition stated vertically. Non-significant differences in taxa relative abundance between conditions are illustrated in Suppl. Figure S3

\section{Supplementary Files}

This is a list of supplementary files associated with this preprint. Click to download.

- FigureS1.pdf

- Figures2.pdf

- FigureS3Carbajal.jpg

- Tables1.csv 